\title{
Designing a Machine Learning-based System to Augment the Work Processes of Medical Secretaries
}

\author{
Patrick S. Johansen, Rune M. Jacobsen, \\ Lukas B. L. Bysted, Mikael B. Skov \\ and Eleftherios Papachristos \\ Department of Computer Science, Aalborg University, Denmark \\ pjohan15@student.aau.dk, rjacob15@student.aau.dk, lbyste15@student.aau.dk \\ dubois@student.aau.dk, papachristos@cs.aau.dk
}

\begin{abstract}
Advances in Machine Learning (ML) provide new opportunities for augmenting work practice. In this paper, we explored how an ML-based suggestion system can augment Danish medical secretaries in their daily tasks of handling patient referrals and allocating patients to a hospital ward. Through a user-centred design process, we studied the work context and processes of two medical secretaries. This generated a model of how a medical secretary would assess a visitation suggestion, and furthermore, it provided insights into how a system could fit into the medical secretaries' daily tasks. We present our system design and discuss how our contribution may be of value to HCI practitioners designing for work augmentation in similar contexts.
\end{abstract}

\section{Keywords}

Work Augmentation $\cdot$ Human-AI Interaction $\cdot$ Medical Domain

How to cite this book chapter:

Johansen, P.S., Jacobsen, R.M., Bysted, L.B.L., Skov, M.B. and Papachristos, E. 2020.

Designing a Machine Learning-based System to Augment the Work Processes of Medical Secretaries. In: Loizides, F., Winckler, M., Chatterjee, U., Abdelnour-Nocera, J. and Parmaxi, A. (eds.) Human Computer Interaction and Emerging Technologies: Adjunct Proceedings from the INTERACT 2019 Workshops. Pp. 191-196. Cardiff: Cardiff University Press. DOI: https://doi.org/10.18573/book3.y. License: CC-BY 4.0. 


\section{Introduction}

The promise of workforce augmentation through artificial intelligence (AI) or machine learning $(\mathrm{ML})$ is the enabling of making professionals more efficient in their work practices. The goal is not to replace workers with fully automated systems but to utilize algorithms to carry out complex or repetitive tasks while high-level decision-making remains under human control. But designing AI-based systems pose significant challenges and is not a straight forward process. A successful system would require a deep understanding of the specific work context in order to be able to answer questions such as: which tasks of the workflow should be automated, and which require human intervention? How can we design in accordance with users' mental models [6]? What information, in what form, and at what time, has to be provided to aid the human decision making process? How can we establish confidence and trust in the systems [4-5]?

In this paper, we investigate and address some of these questions through the design of a system aimed to augment the workflow of medical secretaries (MS) in a hospital. Such systems could potentially make repetitive and administrative tasks more efficient and would free up time and resources that could be used to engage with patients. The system was designed in collaboration with a Danish company providing the system using ML algorithms trained on extensive Danish medical patient data and treatment history. We present our approach to understand and deconstruct the workflow of MS during the visitation process and the methods we used to design the user interface.

\section{Method}

In Denmark, the visitation process begins when a patient is referred from a general practitioner to a hospital. A hospital MS receives the referral and if necessary retrieves additional documentation (e.g. patient records) from various systems. The patient referrals are then preliminary sorted based on assessment and are handed over to the physicians. If no modifications are necessary, the physician appoints medical staff to treatment procedures and returns the documents to the MS, who schedules the treatments. In a booking system, the MS then assigns the appointed treatment to each referral on timeslot and selects the information letters that should be sent to the patient. Finally, the MS confirms the referral booking and checks status in a separate system. The visitation process is an intricate process involving multiple medical professionals and several work subtasks. The purpose of the ML-system is to automate the task of the hospital physicians and provide a suggestion for a visitation that the medical secretary is able to review and process. Each suggestion is based on a wide array of patient data. It is assumed that the augmentation of this process will shorten handling times considerably. 
We conducted three user studies with staff at Aalborg University Hospital in order to deconstruct the workflow and understand the needs and work context of the MS. First, we did a semi-structured interview with one MS at in order to identify hospital staff responsibilities and tasks. Secondly, we conducted a contextual inquiry at the ward to gain insights into the context, environment, and technical details of the visitation process. Thirdly, we constructed a card sorting activity where the MS prioritized, sorted, and ranked visitation information in order for us to understand what cues they are looking for during the decision-making process. Afterwards, findings from the three studies were synthesized in a PACT analysis [3]. Before we present our design, we outline some design implications that emerged from the studies. The semi-structured interviews and contextual inquiry revealed the workflow of the MS and helped generate a list of additional visitation considerations that the medical secretary goes through and controls for when handling visitations (e.g. need to organize transportation or find a translator). It is important that the design is able to convey whether any of those additional considerations apply to a specific visitation suggestion. The bits of information needed to assess a referral were prioritized by the MS in the card sorting activity which proved to be very useful in guiding the design process of the visual user interface.

\section{Design and Evaluation}

A screenshot of the user interface that was developed can be seen in fig. 1. The system consists of two main parts, the visitation suggestion card, and the rest of the system which supports the general workflow of handling visitations. The system provides the MS with a summary of the diagnosis and the cause of referral, providing an overview of the patient in the specific case. This is currently the information that the MS looks for when she builds an understanding of a visitation case. The system also presents an initial suggestion of how the visitation could be handled. This suggestion includes a treatment type, date, time, as well as icons that communicate visitation considerations.

During our studies, we realized that the task of handling visitations is only one of many tasks carried out by the MS, and the process of handling multiple visitations may be interrupted by more urgent tasks. Therefore, we implemented an overview of visitation tasks in a sidebar, where suggestions are sorted in digital stacks according to recommended treatment. This corresponds to the current practice, in which the MS prints the received referrals, she reads them through, highlighting significant information, assigns a preliminary treatment, and sorts the referrals and from there work with the visitation as stacks. In addition, the system implements hourly notifications automatically informing the MS about any acute referrals that have been received during the last hour. This resembles a current work pattern, in which the MS manually checks for incoming referrals and handles them if perceived acute. Finally, if human intervention 


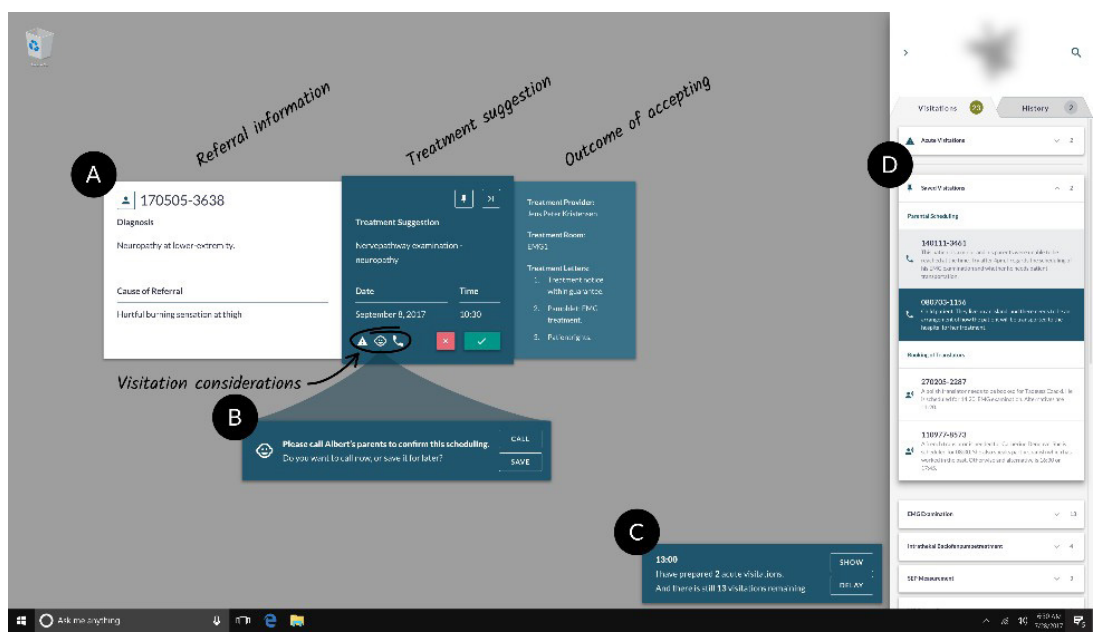

Fig. 1: The picture shows the user interface of our system. A) Visitation suggestion card, B) referral specific notification advising to book a translator for the patient, C) hourly updates about acute referrals, D) Sidebar/Overview. Two panes "Visitation" and "History". Below "Acute Visitations" followed by "Saved Visitations", and lastly categorized referrals by treatment.

is required for a visitation to be completed, the system notifies the MS via a message box (e.g. "This patient might need a translator..." and the phone number to the translators' office). The MS can also save the task for later to schedule the phone calls in bulk.

The design was evaluated by a MS assessing 20 visitation suggestions following the think-aloud protocol. The MS was encouraged to articulate the usefulness of the system as a tool for processing visitations, and furthermore express any concerns regarding use. We purposely created an incorrect visitation suggestion with an erroneous treatment for the patient to test if the MS could properly assess the information of the visitation suggestion, and the MS was able to detect and decline the wrongful suggestion. The result of the evaluation was a list of usability problems and insights on how the MS perceived working with the visitation suggestion system. In the preliminary findings, the MS expressed how the system worked very efficiently and that this, in particular, would free up more time for the MS to talk with patients and focus on patient treatment.

\section{Discussion and Future Work}

While our work is in an early stage, it provides insights from a specific example of how a system can be designed to fit into a complex work context and augment current work practices (e.g. checking for new visitations, notifying). We 
illustrate that it is important, for a system in this context, to be clear about its limitations, and communicating when and what human intervention is needed (e.g. booking translator and contacting under-age patients' parents). In this initial study, we only had access to a limited number of hospital staff but we will further develop our system and generate a stronger empirical foundation by engaging a broader group of medical professionals. Since different hospital departments may have unique challenges with their patient groups, we would expect that other considerations become evident as we change the setting of the system.

The design we propose only provides a static understanding of a suggestion and provides the user with a binary choice of accepting or reject. Abdul et al [1] note that most work on interfaces for intelligibility provides static explanations and that there might be possible to work on allowing users to explore a system interactively. While our system does not focus on explaining the actual AI system, we see the potential for allowing a user to explore elements of a suggestion in a flexible and interactive way (e.g. unfolding the information that a suggestion is based upon). But more importantly, we would like to study the implications of MS using the system over a longer period of time. We are concerned that users might start to rely on the suggestions of a system, introducing what is also known as automation bias [2]. We are especially interested in understanding the relationship between the user's ability to interact with and explore a suggestion, and the degree of complacency that may be introduced over time.

\section{References}

1. Ashraf Abdul, Jo Vermeulen, et al. 2018. Trends and Trajectories for Explainable, Accountable and Intelligible Systems: An HCI Research Agenda. CHI 2018: 1-18.

2. E. Alberdi, P. Ayton, A.A. et al. 2005. Automation bias and system design: a case study in a medical application. IEE and MOD HFI DTC Symposium on People and Systems - Who are we Designing for?, IEE, 53-60.

3. Benyon; David. 2013. PACT: a framework for designing interactive systems. In Designing Interactive Systems. Pearson, 25-48.

4. Monika Hengstler, Ellen Enkel, et al. 2016. Technological Forecasting \& Social Change Applied artificial intelligence and trust-The case of autonomous vehicles and medical assistance devices. Technological Forecasting \& Social Change 105: 105-120.

5. Andreas Holzinger, Markus Plass, et al. 2017. A glass-box interactive machine learning approach for solving NP-hard problems with the human-in-the-loop. 26.

6. Donald A Norman. 1994. How might people interact with agents. CACM 37, 7: 68-71 